\title{
Attitudes of Potential Participants Towards Molecular Therapy Trials in Huntington's Disease
}

\author{
Tanya M. Bardakjian ${ }^{\mathrm{a}, *}$, Kaylee Faulkner Naczi ${ }^{\mathrm{a}}$ and Pedro Gonzalez-Alegre ${ }^{\mathrm{a}, \mathrm{b}}$ \\ ${ }^{a}$ Department of Neurology, Hospital of the University of Pennsylvania, Philadelphia, PA, USA \\ ${ }^{\mathrm{b}}$ Raymond G. Perelman Center for Cellular \& Molecular Therapeutics, The Children's Hospital \\ of Philadelphia, Philadelphia, PA, USA
}

\begin{abstract}
.
Background: Advances in molecular therapeutic approaches in the last decade are translating into the design of non-traditional clinical trials. In order to improve their feasibility, it is important to understand the attitudes of potential participants towards these trials, their motivations to get involved and acceptance of risks.

Objective: We aimed to better understand the willingness of potential participants to participate in different molecular therapy trials for Huntington's disease (HD) based on their clinical and genetic status, trial design and goals of the treatment.

Methods: An anonymous survey was distributed through the Huntington's Disease Society of America (HDSA) on-line portal/website. Various hypothetical scenarios were presented followed by a survey consistent of Likert scale responses ascertaining willingness to participate, collecting demographic, clinical and genetic information.

Results: There were a total of 87 responses, including patients diagnosed with HD, pre-manifesting mutation carriers and asymptomatic participants at risk. The majority of participants indicated they were very likely or likely to participate in clinical trials independent of study design or goals of the therapy, with a more favorable view in premanifesting mutation carriers. However, more invasive procedures and trials including placebo were less favorably viewed across all diagnostic groups.

Conclusions: In summary, most individuals in the HD community would consider participation in novel molecular therapy trials, but study design and goals could impact patient recruitment. This data can be used to inform the recruitment and consent process into clinical trials and to address common concerns by potential participants.
\end{abstract}

Keywords: Huntington's disease, gene therapy, clinical trial

\section{INTRODUCTION}

Huntington's disease (HD) is an autosomal dominant and virtually fully penetrant neurodegenerative disease caused by a triplet repeat expansion in the HTT gene (reviewed by [1]). Clinically, it is

\footnotetext{
${ }^{*}$ Correspondence to: Tanya M. Bardakjian, 330 South Ninth Street, Philadelphia, PA 19107, USA. Tel.: +1 215 829 5159; Fax: +1 215829 3935; E-mail: tanya.bardakjian@uphs.upenn.edu.
}

characterized by the progressive appearance and worsening of motor impairment, abnormal involuntary movements, mainly chorea, psychiatric disease and cognitive impairment. Currently, the only available treatments are symptomatic, aimed at alleviating motor and psychiatric symptoms [2, 3]. Therapeutic development in HD is an area of high priority in translational neuroscience.

Molecular and gene-based therapies are an emerging therapeutic field, with promise for slowing down 
or even curing life-threatening genetic disease. Several studies have addressed the public attitudes and knowledge towards gene therapy [4-6]. Although not specifically addressing neurodegenerative diseases, these studies indicate a limited understanding but an overall positive view towards the use of gene therapy for the treatment of serious illness. The level of accepted risk for a trial likely results from many factors, and researchers and the public in general feel that more severe conditions would tolerate a higher level of risk [7-11]. This view could also be determined by the current status of the participant questioned, from a younger premanifest mutation carrier to an older patient with advanced disease. In HD, clinical trials for symptomatic therapies included symptomatic patients. However, interventions targeting the disease process will likely include initially patients very early in the disease process or even premanifest participants.

Different gene or molecular interventions are under development for HD. For instance, ongoing efforts aim to reduce neuronal levels of expanded huntingtin through repeated intrathecal injections of antisense oligonucleotides (ASOs) [12]. A recent first in human Phase $1 \mathrm{~b} / 2 \mathrm{a}$ clinical trial of IONIS-HTTrx was successfully completed in Europe and Canada utilizing ASOs [13, 14], although the results have not been presented yet in a peer-reviewed manner. A different approach to the injection of Adeno-Associated Virus (AAV) carrying an RNA interference construct to reduce huntingtin levels, offering the possibility of long-lasting clinical benefit with a single major neurosurgical intervention [12, 15-19]. Additional therapeutic approaches targeting HD are under development with the shared goal of interfering with the disease process, such as the monthly intravenous application of anti-semaphorin 4D immunotherapy [20]. It is important to understand how potential participants view these interventions, different from traditional daily tablet or capsule-based oral treatments, ascertain their willingness to participate in trials and understand their concerns. Involving the targeted population in the development of clinical trials could increase compliance with protocols and willingness to enroll [21-23]. A patient-centered trial design, such as through the inclusion of appropriate education and communication to ensure participants and advocates can be informed and engaged, is likely to have a positive impact on recruitment $[5,21,24]$.

In this study, we explore the attitudes of patients at different stages of HD towards different therapeutic interventions, ranging from a daily oral pillbased administration to a single invasive and more risky intervention. We use hypothetical scenario methodology, as previously reported in genetic research [25]. The information collected should be useful for future clinical trial design using molecular interventions in patients with HD and perhaps other genetic disorders.

\section{METHODS}

We designed a questionnaire-based study to assess the attitudes towards gene therapy clinical trials in potential study participants. We included three subgroups of participants: individuals diagnosed with HD, pre-symptomatic mutation carriers and asymptomatic individuals at-risk for HD with no genetic testing. They were recruited through the HDSA on-line portal/website. A survey consistent of mainly Likert scale responses and several multiple choice questions were utilized including hypothetical scenarios (Supplementary Material). Likert-like scales are commonly used in questionnaire-based research, offering a range of answer options from one extreme attitude to another and with a neutral midpoint and can be transformed into numerical values for quantification. We generated hypothetical interventions based on planned or ongoing therapeutic interventions for HD, also including standard oral interventions that most participants are familiar with. For instance, we included a hypothetical intervention in which a pill is taken by mouth once a day. For HD-specific designs, we generated interventions based on repeated intravenous infusions (such as in NCT02481674), intrathecal infusion (based on the infusion of ASOs referenced above, NCT02519036) or single intraparenchymal injections (less well known and, therefore, explained in more detail in the questionnaire, as it would be used in different planned AAV-based trials [12]). The effect of including a placebo arm was also evaluated. We collected basic demographic, clinical and genetic information and stratified participants into diagnosis of HD, pre-manifest known mutation carriers, and at risk participants-not tested. For statistical analysis, each answer was assigned a numerical score as indicated in the figures and legends. ANOVA with Dunnett pot-test comparison of all versus the first group was employed for comparison, using the statistical software GraphPad Prism 5. Data are presented 
as the mean \pm SEM unless otherwise noted. This study was approved by the Institutional Review Committee at the University of Pennsylvania.

\section{RESULTS}

There were a total of 87 participants. Their demographic characteristics and diagnostic status are shown in (Table 1). As shown in (Fig. 1), participants in all diagnostic groups showed a positive attitude towards participation in clinical trials independent of the goals of the study with the exception of premanifest subjects in the case of a high risk trial. Although the total number of pre-manifesting mutation carriers is lower, participants in that group were significantly more positive towards participa-

Table 1

Demographic characteristic of participants

\begin{tabular}{lcccc}
\hline & At Risk & Premanifest & HD & Total \\
\hline N (\%Women) & $33(82)$ & $18(83)$ & $36(58)$ & $87(72)$ \\
Mean Age (SD) & $43.5(2.7)$ & $45.5(3.3)$ & $51.4(1.8)$ & $46.9(1.5)$ \\
Education & & & & \\
$\quad$ GED & 1 & 0 & 3 & 4 \\
$\quad$ High School & 1 & 4 & 5 & 10 \\
College & 27 & 9 & 21 & 57 \\
Graduate & 4 & 5 & 6 & 15 \\
No Response & 0 & 0 & 1 & 1 \\
\hline
\end{tabular}

tion in trials except when higher risk or lower benefit were included. When participants were presented different clinical trial design scenarios, more invasive surgical interventions and the presence of a placebo arm lowered the likelihood to enroll across all diagnostic groups (Fig. 2). Figure 3 shows the participant responses regarding specific factors that could influence the decision to participate in clinical trials. Finally, when asked about their impression of what motivates the investigators conducting these trials, participants clearly believed the main goal is the safety and potential benefit for participants over any financial or career benefits for the researchers (Fig. 4).

\section{DISCUSSION}

In this study, we explore the willingness of participants to participate in novel molecular therapy trials for HD, illustrating how clinical status, study design and goals of the intervention could influence the decision of potential participants to enroll. Moreover, we identify potential drivers for this choice and show the positive impression of potential participants in regards to the motivation of the investigators who conduct these studies. This descriptive study helps us better understand how HD families view the novel
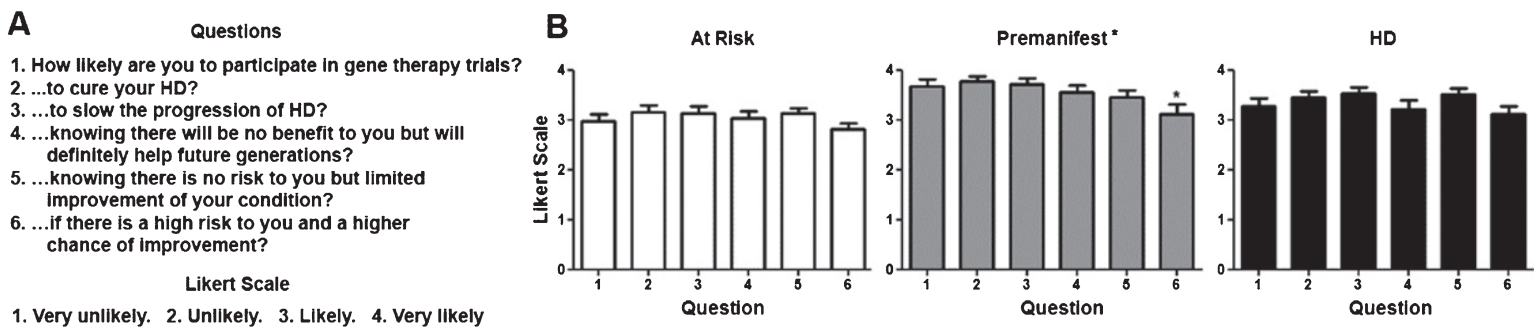

1. Very unlikely. 2. Unlikely. 3. Likely. 4. Very likely

C
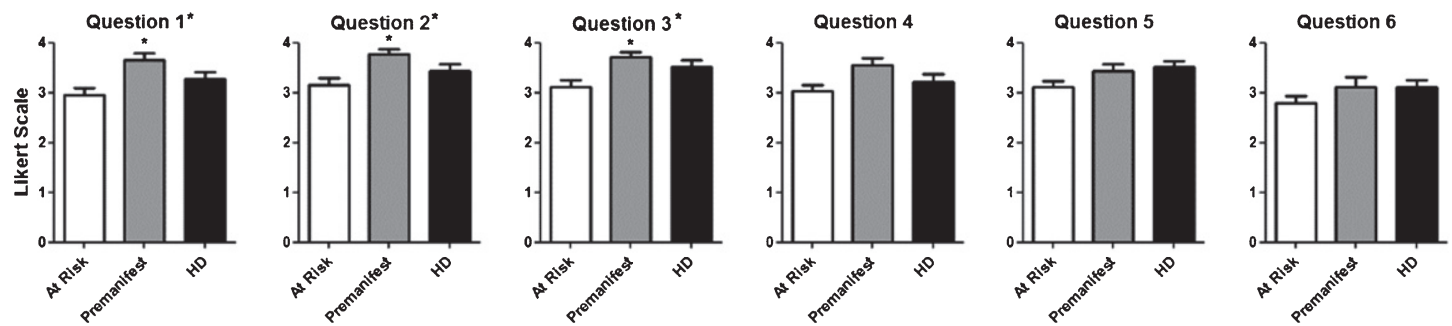

Fig. 1. Willingness to participate in clinical trials based on their objective. A) Questions regarding willingness to participate in trials and Likert scale with potential answers and numerical values assigned to each answer (i.e., 4 for Very Likely, 1 for Very Unlikely). B) Average score for questions per diagnostic group. Numerical values for questions in the $\mathrm{X}$ axis correlate with those shown in (A). C) Average score for each individual question for each. Difference between questions (A) or groups (C) were calculated using ANOVA (when present, significance noted in the title above each graph) with Dunnett pot-test comparison of all versus the first column (when present, significance noted above each column). ${ }^{*} p<0.05$. 

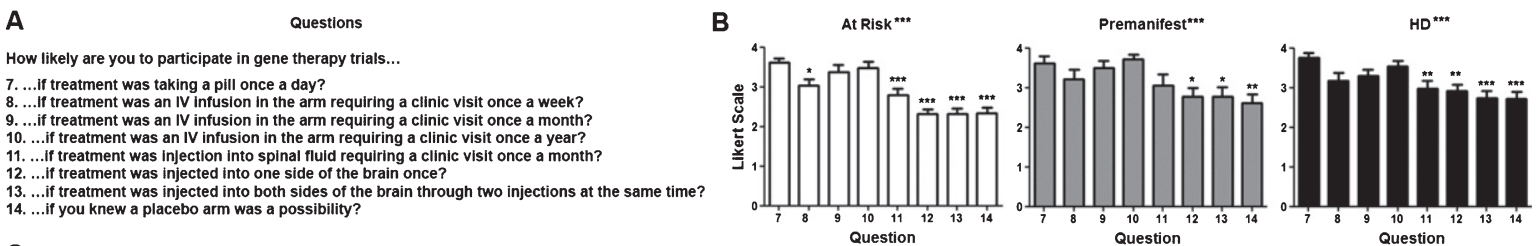

C
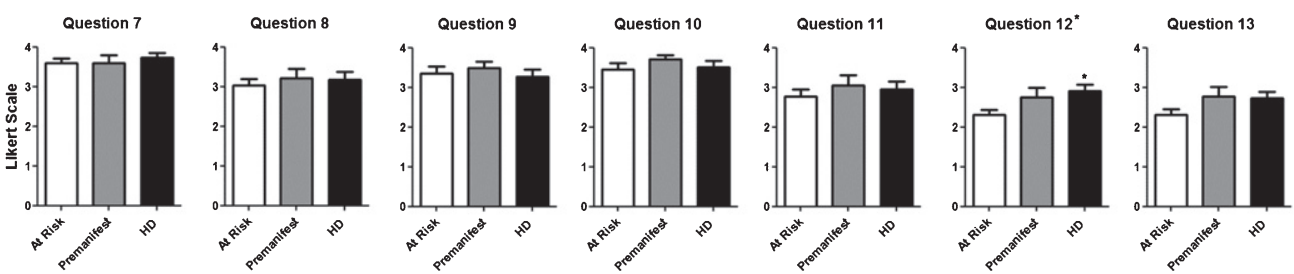

Question 14

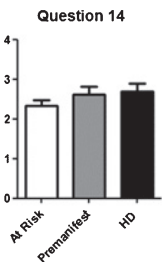

Fig. 2. Willingness to participate in clinical trials based on their design. A) Questions regarding willingness to participate in trials based on the intervention. The same Likert scale as in Fig. 1 was used. B) Average score for questions per diagnostic group. Numerical values for questions in the $\mathrm{X}$ axis correlate with those shown in (A). C) Average score for each individual question for each. Difference between questions (A) or groups (C) were calculated using ANOVA (when present, significance noted in the title above each graph) with Dunnett pot-test comparison of all versus the first column (when present, significance noted above each column). ${ }^{*} p<0.05 ;{ }^{* *} p<0.01 ;{ }^{* * *} p<0.001$.

A

How important are the following factors in your decision to participate in clinical trials?

15. Helping children who are at risk 16. Improving my quality of life

17. Risk to myself

18. Curing HD

19. Helping Science

Likert Scale:

3: Very Important

2: Somewhat Important.

1: Not Important

C
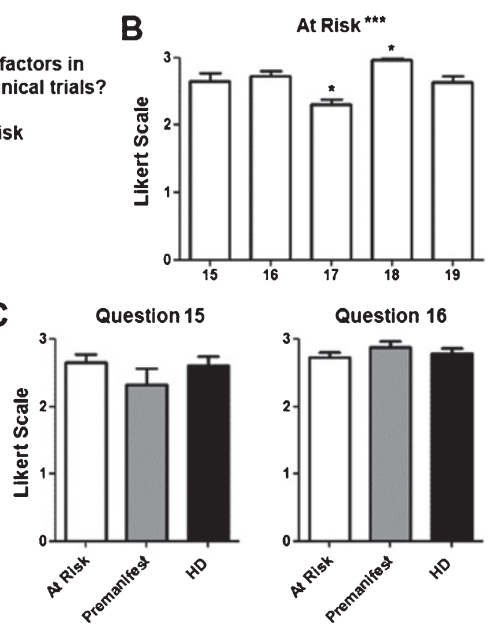
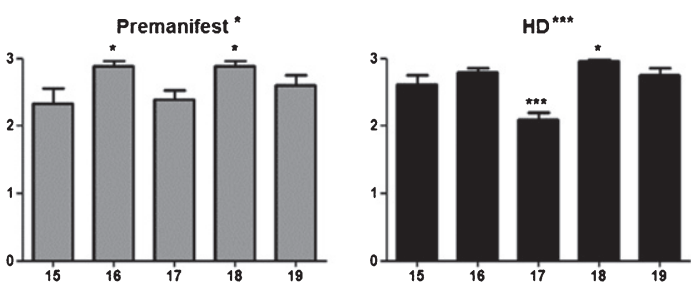

Question 17
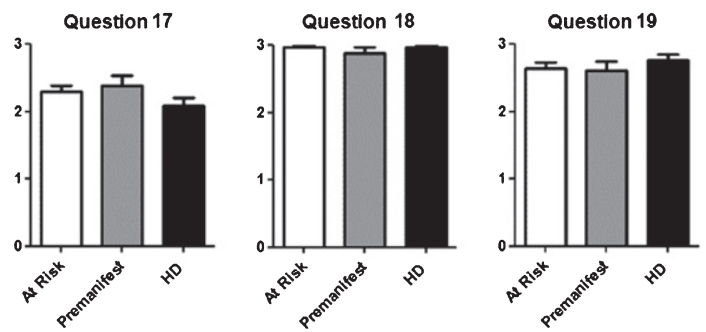

Fig. 3. Factors that influence decision to participate in clinical trials. A) Questions regarding decision to participate in and Likert scale with potential answers and numerical values assigned to each answer. B) Average score for questions per diagnostic group. Numerical values for questions in the $\mathrm{X}$ axis correlate with those shown in (A). C) Average score for each individual question for each. Difference between questions (A) or groups (C) were calculated using ANOVA (when present, significance noted in the title above each graph) with Dunnett pot-test comparison of all versus the first column (when present, significance noted above each column). ${ }^{*} p<0.05 ;{ }^{* *} p<0.01 ;{ }^{* * *} p<0.001$.

therapeutic interventions under development in this field.

We should first emphasize the significant limitations of this study. We acknowledge that this descriptive study has limitations, including a small sample size and highly educated participants. This finding likely indicates that web-based surveys are more likely to reach a more educated population, with more access to and familiarity with internet navigation. This shortcoming of this study suggests that the increasing use of novel technologies and web-based tools for recruitment to clinical trials could have similar consequences, resulting in skewed cohorts of study participants that might not represent the entire HD population. In addition, this questionnaire was not previously validated and, despite the majority of respondents being at least college-educated, we could not verify their degree of understanding of the questions asked. Also, a long survey with different scenarios that require comprehension could discourage more cognitively impaired participants from participating, introducing another potential 
A

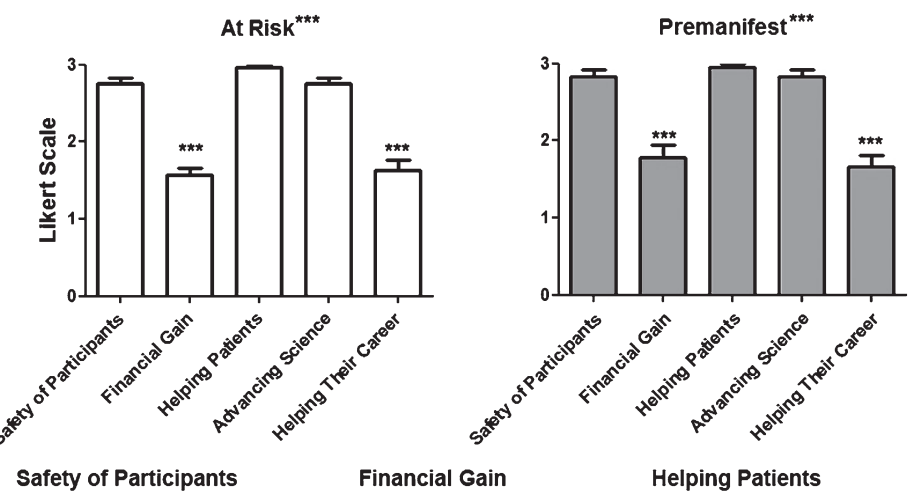

B
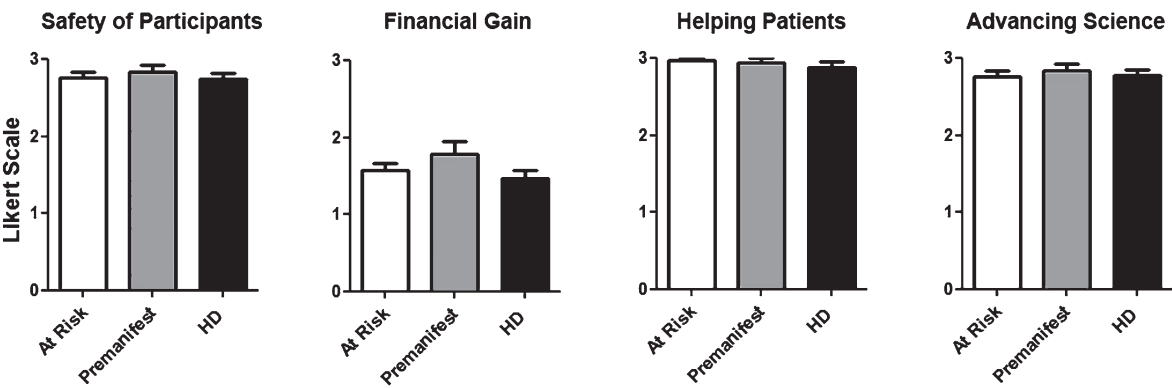

$H D^{* * *}$

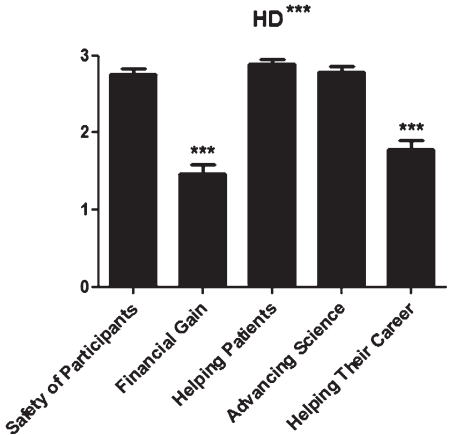

Fig. 4. How participants perceive the investigators' motivation to conduct trials. Questions were asked regarding the participant's impression of the researcher motivations to conduct clinical trials. The same Likert scale as in Fig. 3 was used. A) Average score for questions per diagnostic group. B) Average score for each individual question for each. Difference between questions (A) or groups (B) were calculated using ANOVA (when present, significance noted in the title above each graph) with Dunnett pot-test comparison of all versus the first column (when present, significance noted above each column). ${ }^{*} p<0.05 ;{ }^{* *} p<0.01 ;{ }^{* * *} p<0.001$.

source of selection bias. Nevertheless, our results are in agreement with the subjective opinions we receive from HD patients and families during clinical interactions. We believe this information is helpful for the design of trials with novel therapeutic modalities.

The understanding of the public about the genetics of neurological diseases [26] or the attitudes of researchers towards gene therapy trials [8] have been evaluated, but there is a paucity of studies addressing the attitude of potential participants with neurodegenerative diseases for these trials. Overall, participants in our study expressed a higher likelihood to participate in gene therapy trials if they treatment was less invasive and less frequent, as expected. Not surprisingly, compared with the option of an oral medication, IV infusion were acceptable but willingness to participate gradually decreased with intrathecal injections or an intraparenchymal injection. Nevertheless, even in the case of the most invasive procedure, the participants still provided a positive view towards participation. Consistent with previous studies [27, 28], the negative effects of including a placebo group in the likelihood to participate was comparable to the most invasive intervention. This finding stresses the need to continue educating potential participants on the role of a placebo arm, and providing the option of receiving active treatment after the placebo period in future trials.

An important question in the context of gene therapies for neurodegenerative diseases is the timing of the intervention. For huntingtin lowering therapies, the treatment would be ideally applied prior to the onset of symptoms or in the very early stages $[1,2,12]$. Participants need to understand the natural history of the condition and the scientific reasons why treatment must be geared towards early disease, if not before the manifestation of symptoms. We hypothesized that individuals would be less inclined to volunteer for research studies when their disease has not fully manifested. However, premanifest mutation carriers expressed a higher willingness to enroll in trials than at risk (not tested) asymptomatic participants, except when interventions carry a higher risk of less potential benefit. This finding might reflect a testing bias, however, as participants at risk be could less motivated to pursue predictive testing if they are less inclined to participate in a clinical trial. Nevertheless, Grill et al. [29] also found a high willingness 
to enroll in clinical trials for participants in "preclinical" stages of Alzheimer's disease. Together with our data, that study highlights the high level of interest of participants at risk for neurodegenerative disease to enroll in innovative therapeutic trials.

It is not surprising that a key driver of motivation to participate in clinical trials are finding a cure for HD and improve the quality of life of participants. The fact that the inherent risk of receiving an investigational treatment is less important and the possibility of placebo more important indicates that potential participants are more focused on the efficacy than safety of the investigational agent. Our findings indicate a high degree of trust of potential participants on the motivations guiding researchers who conduct these trials, believing that the medical community works towards a cure for the benefit of patients over personal or professional gain.

In conclusion, although with important limitations as discussed above, this study suggests that the HD population has a very high willingness to participate in gene therapy trials. However, study design and the disease stage of the subject even when invasive and perceived as "risky". Pre-manifesting mutation carriers, a target for upcoming trials, appear more enthusiastic about participation.

\section{ACKNOWLEDGMENTS}

This study was supported by a grant from the Personalized Medicine Special Interest group of the National Society of Genetic Counselors to Tanya Bardakjian. We thank all the participants for their participation and the HDSA for distributing the survey and Dr. Jeff Long (University of Iowa) for advice in statistical analysis.

\section{CONFLICT OF INTEREST}

Tanya Bardakjian and Kaylee Faulkner have no conflict of interest to report. Pedro Gonzalez-Alegre received consulting fees from Teva and Spark Therapeutics.

\section{SUPPLEMENTARY MATERIAL}

The supplementary material is available in the electronic version of this article: http://dx.doi.org/ 10.3233/JHD-180328.

\section{REFERENCES}

[1] Ross CA, Aylward EH, Wild EJ, Langbehn DR, Long JD, Warner JH, et al. Huntington disease: Natural history, biomarkers and prospects for therapeutics. Nat Rev Neurol. 2014;10(4):204-16

[2] Bates GP, Dorsey R, Gusella JF, Hayden MR, Kay C, Leavitt BR, et al. Huntington disease. Nat Rev Dis Primers. 2015;1:15005.

[3] Coppen EM, Roos RA. Current pharmacological approaches to reduce chorea in Huntington's disease. Drugs. 2017;77(1):29-46.

[4] Iredale R, Dolan G, McDonald K, Kirk M. Public attitudes to human gene therapy: A Pilot study in Wales. Community Genet. 2003;6(3):139-46.

[5] Strong H, Mitchell MJ, Goldstein-Leever A, Shook L, Malik P, Crosby LE. Patient perspectives on gene transfer therapy for sickle cell disease. Adv Ther. 2017;34(8):2007-21.

[6] Wang JH, Wang R, Lee JH, Iao TWU, Hu X, Wang YM, et al. Public attitudes toward gene therapy in China. Mol Ther Methods Clin Dev. 2017;6:40-2.

[7] Calnan M, Montaner D, Horne R. How acceptable are innovative health-care technologies? A Survey of public beliefs and attitudes in England and Wales. Soc Sci Med. 2005;60(9): 1937-48.

[8] Deakin CT, Alexander IE, Hooker CA, Kerridge IH. Gene therapy researchers' assessments of risks and perceptions of risk acceptability in clinical trials. Mol Ther. 2013;21(4):806-15.

[9] Hudson J, Orviska M. European attitudes to gene therapy and pharmacogenetics. Drug Discov Today. 2011;16(1920):843-7.

[10] Macer DR, Akiyama S, Alora AT, Asada Y, Azariah J, Azariah H, et al. International perceptions and approval of gene therapy. Hum Gene Ther. 1995;6(6):791-803.

[11] Robillard JM, Roskams-Edris D, Kuzeljevic B, Illes J. Prevailing public perceptions of the ethics of gene therapy. Hum Gene Ther. 2014;25(8):740-6.

[12] Wild EJ, Tabrizi SJ. Therapies targeting DNA and RNA in Huntington's disease. Lancet Neurol. 2017;16(10):837-47.

[13] Rodrigues FB, Wild EJ. Huntington's disease clinical trials corner: February 2018. J Huntingtons Dis. 2018;7(1):89-98.

[14] van Roon-Mom WMC, Roos RAC, de Bot ST. Dosedependent lowering of mutant huntingtin using antisense oligonucleotides in Huntington disease patients. Nucleic Acid Ther. 2018;28(2):59-62.

[15] Maguire CA, Ramirez SH, Merkel SF, Sena-Esteves M, Breakefield XO. Gene therapy for the nervous system: Challenges and new strategies. Neurotherapeutics. 2014;11(4): 817-39.

[16] Dunbar CE, High KA, Joung JK, Kohn DB, Ozawa K, Sadelain M. Gene therapy comes of age. Science. 2018; 359(6372).

[17] Pfister EL, DiNardo N, Mondo E, Borel F, Conroy F, Fraser $\mathrm{C}$, et al. Artificial miRNAs reduce human mutant huntingtin throughout the striatum in a transgenic sheep model of Huntington's disease. Hum Gene Ther. 2018;29(6):663-73.

[18] McBride JL, Clark RL. Stereotaxic surgical targeting of the nonhuman primate caudate and putamen: Gene therapy for Huntington's disease. Methods Mol Biol. 2016;1382: 409-28.

[19] McBride JL, Pitzer MR, Boudreau RL, Dufour B, Hobbs T, Ojeda SR, et al. Preclinical safety of RNAi-mediated HTT suppression in the rhesus macaque as a potential therapy for Huntington's disease. Mol Ther. 2011;19(12):2152-62. 
[20] Southwell AL, Franciosi S, Villanueva EB, Xie Y, Winter LA, Veeraraghavan J, et al. Anti-semaphorin 4D immunotherapy ameliorates neuropathology and some cognitive impairment in the YAC128 mouse model of Huntington disease. Neurobiol Dis. 2015;76:46-56.

[21] Mullins CD, Vandigo J, Zheng Z, Wicks P. Patientcenteredness in the design of clinical trials. Value Health. 2014;17(4):471-5.

[22] Purks JL, Wilhelm EE, Shoulson I, Creveling J, Dorsey ER, Irony $\mathrm{T}$, et al. Inaugural conference on incorporating patientreported outcomes and patient preference information into clinical research, clinical care, and risk-benefit assessments for neurodegenerative diseases. Patient. 2017;10(5):541-4.

[23] Wicks P, Vaughan T, Heywood J. Subjects no more: What happens when trial participants realize they hold the power? BMJ. 2014;348:g368.

[24] Gottweis H. Gene therapy and the public: A Matter of trust. Gene Ther. 2002;9(11):667-9.
[25] Persky S, Kaphingst KA, Condit CM, McBride CM. Assessing hypothetical scenario methodology in genetic susceptibility testing analog studies: A Quantitative review. Genet Med. 2007;9(11):727-38.

[26] Stockdale A. Public understanding of genetics and Alzheimer disease. Genet Test. 1999;3(1):139-45.

[27] Avenell A, Grant AM, McGee M, McPherson G, Campbell MK, McGee MA, et al. The Effects of an open design on trial participant recruitment, compliance and retention-a randomized controlled trial comparison with a blinded, placebo-controlled design. Clin Trials. 2004;1(6):490-8.

[28] Welton AJ, Vickers MR, Cooper JA, Meade TW, Marteau TM. Is recruitment more difficult with a placebo arm in randomised controlled trials? A Quasirandomised, interview based study. BMJ. 1999;318(7191):1114-7.

[29] Grill JD, Karlawish J, Elashoff D, Vickrey BG. Risk disclosure and preclinical Alzheimer's disease clinical trial enrollment. Alzheimers Dement. 2013;9(3):356-9 e1. 